ESAIM: COCV 21 (2015) 372-377

DOI: $10.1051 / \mathrm{cocv} / 2014022$
ESAIM: Control, Optimisation and Calculus of Variations

www.esaim-cocv.org

\title{
FRANK ENERGY FOR NEMATIC ELASTOMERS: A NONLINEAR MODEL
}

\author{
Marco Barchiesi ${ }^{1}$ And Antonio DeSimone ${ }^{2}$
}

\begin{abstract}
We discuss the well-posedness of a new nonlinear model for nematic elastomers. The main novelty in our work is that the Frank energy penalizes spatial variations of the nematic director in the deformed, rather than in the reference configuration, as it is natural in the case of large deformations.
\end{abstract}

Mathematics Subject Classification. 74B20, 74G65.

Received August 30, 2013.

Published online November 27, 2014.

In this paper we discuss a new nonlinear model for nematic elastomers, which contains a Frank energy term penalizing spatial variations of the nematic director $\boldsymbol{n}$ in the deformed configuration. Our main result is a theorem on the existence of energy minimizers, which identifies a class of energy densities for which the model is mathematically well-posed.

In recent years, considerable attention has been devoted to fully nonlinear mechanical models describing the coupling between elasticity and nematic order (see $[1,2,6,7,10,16]$ and references therein). The Frank term, however, has been typically evaluated in the reference configuration while, when large deformations are in order, it is more natural to consider spatial variations in the deformed configuration. Our main result is precisely that of establishing (under reasonable assumptions) existence of minimal energy states when the Frank term is written in the deformed configuration.

From the mathematical point of view, the difficulty we face is that our energy functional has two terms, the Frank one defined on the deformed configuration, and the mechanical one defined on the reference configuration. Therefore, we need to push-forward the second one in order to work on the same domain. For this task, it becomes necessary to work with the inverse of the deformation mapping and establishing sufficient regularity properties of this inverse map using only the natural energy bounds is problematic.

Let $\mathcal{M}:=\left\{F \in \mathbb{R}^{3 \times 3}: \operatorname{det} F=1\right\}$ and let $W: \mathcal{M} \rightarrow[0, \infty)$ be a function such that $\widetilde{W}(F):=W\left(F F^{T}\right)$ is polyconvex and $W(F)=0$ if and only if $F=I$. We follow [1,10] and we consider an energy density $W_{\text {mec }}: \mathcal{M} \times \mathbb{S}^{2} \rightarrow[0, \infty)$ defined by

$$
W_{\mathrm{mec}}(F, \boldsymbol{n}):=\widetilde{W}\left(V_{\boldsymbol{n}}^{-1} F\right),
$$

Keywords and phrases. Nematic elastomers, polyconvexity, invertibility.

1 Università degli Studi di Napoli "Federico II", Via Cintia, 80126 Napoli, Italy. barchies@gmail.com

2 SISSA-International School for Advanced Studies, Via Bonomea 265, 34136 Trieste, Italy. desimone@sissa.it 
where $V_{\boldsymbol{n}}$ is the stretch in the direction $\boldsymbol{n} \in \mathbb{S}^{2}$ of a fixed amplitude $\alpha>0$ :

$$
V_{\boldsymbol{n}}:=\alpha \boldsymbol{n} \otimes \boldsymbol{n}+(I-\boldsymbol{n} \otimes \boldsymbol{n}) / \sqrt{\alpha} .
$$

Observe that $W_{\text {mec }}(F, \boldsymbol{n})=0$ implies $V_{n}^{-1} F\left(V_{n}^{-1} F\right)^{T}=I$ and so, since $V_{n}^{-1}$ is symmetric, $F F^{T}=V_{n}^{2}$. In particular, since $\operatorname{det} F=1$, by polar decomposition it follows that $F=V_{n} R$ for some $R \in S O(3)$. This last equality summarizes in one formula the main features of the coupling between deformation $F$ and nematic order $\boldsymbol{n}$ envisaged in [16], showing that every pair $\left(V_{\boldsymbol{n}} R, \boldsymbol{n}\right)$ is a 'natural' (or 'stress-free') state of the material.

We propose the following type of energy to describe nematic elastomers:

$$
\mathcal{I}(u, \boldsymbol{n}):=\int_{u(\Omega)}|\nabla \boldsymbol{n}(y)|^{2} \mathrm{~d} y+\int_{\Omega} W_{\operatorname{mec}}(\nabla u(x), \boldsymbol{n}(u(x))) \mathrm{d} x,
$$

where $u: \Omega \rightarrow \mathbb{R}^{3}$ is a deformation of a body whose reference configuration is $\Omega$ (a bounded, connected, open subset of $\mathbb{R}^{3}$ ) and $\boldsymbol{n}: u(\Omega) \rightarrow \mathbb{S}^{2}$ is the director field describing the nematic order in the elastomer. Note that the gradient operator in the first integral is meant with respect to the current spatial variable $y$, while the gradient in the second term is with respect to the material coordinate $x$. In what follows, $\mathcal{I}_{\text {nem }}(u, \boldsymbol{n})=\int_{u(\Omega)}|\nabla \boldsymbol{n}|^{2} \mathrm{~d} y$ and $\mathcal{I}_{\text {mec }}(u, \boldsymbol{n})=\int_{\Omega} W_{\text {mec }}(\nabla u, \boldsymbol{n} \circ u) \mathrm{d} x$ will denote the nematic (or Frank) and the mechanical term of our energy, respectively. As already highlighted, the main feature (and novelty) of our model is that it is formulated in the deformed configuration rather than in the reference one.

Let us introduce the ambient space of our problem, namely, the class of competitors we allow when minimizing energy (0.1). We assume that the deformations belong to

$$
\mathcal{W}\left(\Omega, \mathbb{R}^{3}\right):=\left\{u \in W^{1,3}\left(\Omega, \mathbb{R}^{3}\right): \operatorname{det} \nabla u=1 \text { a.e. in } \Omega\right\} .
$$

A function $u \in \mathcal{W}\left(\Omega, \mathbb{R}^{3}\right.$ ) has nice properties (see [12]): firstly, it is continuous and differentiable a.e. in $\Omega$. Moreover, it satisfies the $N$ property $(|u(D)|=0$ whenever $D \subset \Omega$ is a measurable set such that $|D|=0)$, and the $N^{-1}$ property $\left(\left|u^{-1}(D)\right|=0\right.$ whenever $D \subset \mathbb{R}^{3}$ is a measurable set such that $\left.|D|=0\right)$.

Let $\Omega_{u}:=\{x \in \Omega: u$ is differentiable in $x\}$. By [11], Theorem 3.1, $u$ is almost locally invertible in $\Omega_{u}$ : for every $x_{0} \in \Omega_{u}$ there are $r=r\left(x_{0}\right)>0$, an open neighborhood $O \subset \Omega$ of $x_{0}$, and a function $w \in W^{1,1}\left(B_{r}\left(y_{0}\right), \mathbb{R}^{3}\right)$ (with $\left.y_{0}=u\left(x_{0}\right)\right)$ such that

$$
\begin{aligned}
& u(O)=B_{r}\left(y_{0}\right) \text { and } w \circ u(x)=x \text { a.e. } x \in O ; \\
& w\left(B_{r}\left(y_{0}\right)\right)=O \text { a.e. and } u \circ w(y)=y \text { for every } y \in B_{r}\left(y_{0}\right) \\
& \nabla w(y)=(\nabla u)^{-1}(w(y)) \text { a.e. } y \in B_{r}\left(y_{0}\right) .
\end{aligned}
$$

Note that, if $[u(\Omega)]$ denotes the interior of $u(\Omega)$, then $u\left(\Omega_{u}\right) \subset[u(\Omega)]$ and therefore, by the $N$ property, $|u(\Omega) \backslash[u(\Omega)]|=0$.

Remark 1. In general a map $u \in \mathcal{W}\left(\Omega, \mathbb{R}^{3}\right)$ could be not open, as shown in Example 1 from [5], by considering the cylinder $\Omega:=\left\{x \in \mathbb{R}^{3}: 0 \leq R<1,\left|x_{3}\right|<1\right\}$, with $R:=\left(x_{1}^{2}+x_{2}^{2}\right)^{1 / 2}$, and the deformation $u(x):=$ $2^{1 / 3}\left(R^{-1 / 2} x_{1}, R^{-1 / 2} x_{2}, R x_{3}\right)$. Since $u$ maps the axis of the cylinder into the origin, $u(\Omega)$ is not open.

Regarding the director field $\boldsymbol{n}$, we assume that, given a deformation $u$, it belongs to $H^{1}\left([u(\Omega)], \mathbb{S}^{2}\right)$. Note that, since $\boldsymbol{n}$ is measurable and $u$ is continuous, the composition $\boldsymbol{n} \circ u$ is measurable. Moreover, since $u$ has the $N^{-1}$ property, this composition does not depend on the particular representative of $\boldsymbol{n}$. Therefore, the mechanical part of our energy is well defined.

Let us make some comments about our ambient space. The stored energy density we have in mind is the standard one describing incompressible Ogden materials $[1,10]$ :

$$
\widetilde{W}(F):=\sum_{i} a_{1}\left(v_{1}^{\alpha_{i}}+v_{2}^{\alpha_{i}}+v_{3}^{\alpha_{i}}-3\right)+\sum_{j} b_{1}\left(\left(v_{1} v_{2}\right)^{\beta_{j}}+\left(v_{1} v_{3}\right)^{\beta_{j}}+\left(v_{2} v_{3}\right)^{\beta_{j}}-3\right),
$$


where $v_{k}=v_{k}(F)$ are the singular values of $F, a_{i}>0, \alpha_{i} \geq 1, b_{j}>0, \beta_{j} \geq 1$, and the normalizing constant $3=\operatorname{tr} I$ is added so that $\widetilde{W}$ vanishes when $F F^{T}=I$. By [8], Theorem 4.9-2, we know that on $\mathcal{M}$ such energy is polyconvex and satisfies a coerciveness inequality of the form

$$
\widetilde{W}(F) \geq a|F|^{\alpha}+b|\operatorname{cof} F|^{\beta}-c,
$$

for suitable $a, b, c>0$ and with $\alpha=\max _{i}\left\{\alpha_{i}\right\}, \beta=\max _{j}\left\{\beta_{j}\right\}$. At the moment our existence result is limited to the case $\alpha=3$, so that the right ambient space is $\mathcal{W}\left(\Omega, \mathbb{R}^{3}\right)$. Indeed we need a certain regularity not only on the deformation $u$, but also on its "inverse" $u^{-1}$. Of course it should be desirable to extend the result to weaker coercivity assumptions already considered in nonlinear elasticity, such as $\alpha=2$ and $\beta=3 / 2$ (see [4,15]), but this goal seems hard to achieve. It would also be interesting to formulate our model in the setting introduced in $[13,14]$, namely, a variational model that allows for cavitation, through a functional that measures in the deformed configuration the surface area of the cavities opened by the deformation.

In order to prove the existence of minimizers in our model, we need a couple of ingredients. The first one is a stability result about invertibilty in the space $\mathcal{W}\left(\Omega, \mathbb{R}^{3}\right)$.

Lemma 1. Let $u, u_{k} \in \mathcal{W}\left(\Omega, \mathbb{R}^{3}\right)$ be such that $u_{k} \rightarrow u$ in $W^{1,3}$ and let $\Omega^{\prime}$ be an open set compactly included in $\Omega$. Then there exists a subsequence of $\left\{u_{k}\right\}$ (not relabeled) such that $u_{k}$ converges to u uniformly in $\bar{\Omega}^{\prime}$. Moreover, for any $x_{0} \in \Omega_{u}^{\prime}$ there exist open neighborhoods $O, O_{k} \subset \Omega^{\prime}$ of $x_{0}, k_{0} \in \mathbb{N}, r=r\left(x_{0}\right)>0$, and $w, w_{k}: B_{r}\left(y_{0}\right) \rightarrow \mathbb{R}^{3}$ with $y_{0}=u\left(x_{0}\right)$ such that for $k \geq k_{0}$

- $u(O)=B_{r}\left(y_{0}\right)$ and $w \circ u(x)=x$ a.e. $x \in O$;

- $u_{k}\left(O_{k}\right)=B_{r}\left(y_{0}\right)$ and $w_{k} \circ u_{k}(x)=x$ a.e. $x \in O_{k}$;

- $u \circ w(y)=y$ and $\nabla w(y)=(\nabla u)^{-1}(w(y))$ a.e. $y \in B_{r}\left(y_{0}\right)$;

- $u_{k} \circ w_{k}(y)=y$ and $\nabla w_{k}(y)=\left(\nabla u_{k}\right)^{-1}\left(w_{k}(y)\right)$ a.e. $y \in B_{r}\left(y_{0}\right)$;

- $\inf \left\{\operatorname{diam}\left(w\left(B_{s}\left(y_{0}\right)\right)\right): s \leq r\right\}=0$;

- $\chi_{O_{k}} \rightarrow \chi_{O}$ pointwise a.e.;

- $w, w_{k} \in W^{1, \frac{3}{2}}\left(B_{r}\left(y_{0}\right), \mathbb{R}^{3}\right)$ and $w_{k} \rightarrow w$ in $W^{1, \frac{3}{2}}$;

- $\operatorname{cof} \nabla w, \operatorname{cof} \nabla w_{k} \in L^{3}\left(B_{r}\left(y_{0}\right), \mathbb{R}^{3 \times 3}\right)$ and $\operatorname{cof} \nabla w_{k} \rightarrow \operatorname{cof} \nabla w$ in $L^{3}$.

Proof. With the exception of the last point, this lemma can be obtained, Lemmas 4.3 and 4.5 from [11]. Remember that if $F \in \mathcal{M}$, then $\operatorname{cof} F^{T}=F^{-1}$. By a change of variables (see [11], Lems. 2.4 and 3.5) we have

$$
\int_{B_{r}\left(y_{0}\right)}\left|\operatorname{cof} \nabla w_{k}\right|^{3} \mathrm{~d} y=\int_{B_{r}\left(y_{0}\right)}\left|\left(\nabla w_{k}\right)^{-1}\right|^{3} \mathrm{~d} y=\int_{O_{k}}\left|\nabla u_{k}\right|^{3} \mathrm{~d} x
$$

so that $\left\{\operatorname{cof} \nabla w_{k}\right\}$ is bounded in $L^{3}$. Similar arguments show that $\operatorname{cof} \nabla w$ belongs to $L^{3}$. In order to prove weak convergence, we still make use of a change of variables. Indeed, because of the low integrability of $\nabla w_{k}$, we cannot appeal to the usual continuity of the cofactor ([9], Thm. 8.20). Let $\phi \in C_{0}^{\infty}\left(B_{r}\left(y_{0}\right)\right)$. Note that $\chi_{O_{k}} \phi \circ u_{k}$ converges to $\chi_{O} \phi \circ u$ pointwise a.e. and therefore in $L^{p}$ for any $p$ finite (by Vitali convergence theorem, being bounded in $\left.L^{\infty}\right)$. We have

$$
\begin{aligned}
\lim _{k} \int_{B_{r}\left(y_{0}\right)} \phi(y) \operatorname{cof} \nabla w_{k}(y) \mathrm{d} y & =\lim _{k} \int_{O_{k}} \phi\left(u_{k}(x)\right)\left(\nabla u_{k}(x)\right)^{T} \mathrm{~d} x \\
& =\int_{O} \phi(u(x))(\nabla u(x))^{T} \mathrm{~d} x \\
& =\int_{B_{r}\left(y_{0}\right)} \phi(y) \operatorname{cof} \nabla w(y) \mathrm{d} y .
\end{aligned}
$$

The second ingredient is the continuity of the cofactor of the "perturbed" gradient $\nabla w V_{\boldsymbol{n}}$ with respect to weak convergence. 
Lemma 2. Let $B$ be a bounded open subset of $\mathbb{R}^{3}$, and let $\left\{w_{k}\right\} \subset W^{1, \frac{3}{2}}\left(B, \mathbb{R}^{3}\right)$ and $\left\{\boldsymbol{n}_{k}\right\} \subset L^{\infty}\left(B, \mathbb{S}^{2}\right)$ be two sequences such that $w_{k} \rightarrow w$ weakly in $W^{1, \frac{3}{2}}, \operatorname{cof} \nabla w_{k} \rightarrow \operatorname{cof} \nabla w$ weakly in $L^{3}$, and $\boldsymbol{n}_{k} \rightarrow \boldsymbol{n}$ pointwise a.e. Then $\nabla w_{k} V_{\boldsymbol{n}_{k}} \rightarrow \nabla w V_{\boldsymbol{n}}$ weakly in $L^{\frac{3}{2}}$ and $\operatorname{cof}\left(\nabla w_{k} V_{\boldsymbol{n}_{k}}\right) \rightarrow \operatorname{cof}\left(\nabla w V_{\boldsymbol{n}}\right)$ weakly in $L^{3}$.

Proof. First of all, note that $\boldsymbol{n} \in L^{\infty}\left(B, \mathbb{S}^{2}\right)$. Moreover $V_{\boldsymbol{n}_{k}} \rightarrow V_{\boldsymbol{n}}$ and $V_{\boldsymbol{n}_{k}}^{-1} \rightarrow V_{\boldsymbol{n}}^{-1}$ pointwise a.e.. On the other hand, since $\left\{V_{\boldsymbol{n}_{k}}\right\}$ and $\left\{V_{\boldsymbol{n}_{k}}^{-1}\right\}$ are both bonded in $L^{\infty}$, Vitali convergence theorem leads to $V_{\boldsymbol{n}_{k}} \rightarrow V_{\boldsymbol{n}}$ and $V_{\boldsymbol{n}_{k}}^{-1} \rightarrow V_{\boldsymbol{n}}^{-1}$ strongly in any $L^{p}, p$ finite. This directly implies that $\nabla w_{k} V_{\boldsymbol{n}_{k}} \rightarrow \nabla w V_{\boldsymbol{n}}$ weakly in $L^{1}$, and then in $L^{\frac{3}{2}}$ because there $\left\{\nabla w_{k} V_{\boldsymbol{n}_{k}}\right\}$ is bounded. Similarly

$$
\operatorname{cof}\left(\nabla w_{k} V_{\boldsymbol{n}_{k}}\right)=\left(V_{\boldsymbol{n}_{k}}\right)^{-1}\left(\operatorname{cof} \nabla w_{k}\right) \rightarrow\left(V_{\boldsymbol{n}}\right)^{-1}(\operatorname{cof} \nabla w)=\operatorname{cof}\left(\nabla w V_{\boldsymbol{n}}\right) \text { weakly in } L^{3} .
$$

We are now ready to prove our main result.

Theorem 1. Assume that $\widetilde{W}$ satisfies the following coercivity condition:

$$
\widetilde{W}(F) \geq c_{1}|F|^{3}-c_{2} \quad \forall F \in \mathcal{M}
$$

for some constants $c_{1}, c_{2}>0$. Assume also that $\Omega$ has smooth boundary and let $\Gamma \neq \emptyset$ be an open (in the relative topology) subset of $\partial \Omega$. Given $\left(u_{0}, \boldsymbol{n}_{0}\right) \in \mathcal{W}\left(\Omega, \mathbb{R}^{3}\right) \times H^{1}\left(\left[u_{0}(\Omega)\right], \mathbb{S}^{2}\right)$ such that $\mathcal{I}\left(u_{0}, \boldsymbol{n}_{0}\right)$ is finite, define $\mathcal{W}_{\Gamma, u_{0}}\left(\Omega, \mathbb{R}^{3}\right):=\left\{u \in \mathcal{W}\left(\Omega, \mathbb{R}^{3}\right): u=u_{0}\right.$ on $\left.\Gamma\right\}$ (the equality is intended in the sense of traces). Then, there exists $(u, \boldsymbol{n}) \in \mathcal{W}_{\Gamma, u_{0}}\left(\Omega, \mathbb{R}^{3}\right) \times H^{1}\left([u(\Omega)], \mathbb{S}^{2}\right)$ minimizing $\mathcal{I}$.

Proof. We are going to use the direct method of the calculus of variations. Let $\left\{\left(u_{k}, \boldsymbol{n}_{k}\right)\right\} \subset \mathcal{W}_{\Gamma, u_{0}}\left(\Omega, \mathbb{R}^{3}\right) \times$ $H^{1}\left(\left[u_{k}(\Omega)\right], \mathbb{S}^{2}\right)$ be a minimizing sequence. Since $F\left(u_{k}, \boldsymbol{n}_{k}\right) \leq F\left(u_{0}, \boldsymbol{n}_{0}\right)$, and $\left\{\left(V_{\boldsymbol{n}_{k}}\right)^{-1}\right\}$ is bounded in $L^{\infty}$, assumption (0.2) implies that $\left\{\nabla u_{k}\right\}$ is bounded in $L^{3}$. Moreover, thanks to the boundary condition and the Poincaré inequality, $\left\{u_{k}\right\}$ is bounded in $L^{3}$. Therefore, by refining the sequence if necessary, we have that $u_{k}$ converges weakly in $W^{1,3}$ to a certain $u$. The continuity of the determinant (see [9], Thm. 8.20) ensures that $\operatorname{det} \nabla u_{k} \rightarrow \operatorname{det} \nabla u$ in distribution and then that $\operatorname{det} \nabla u=1$ a.e. in $\Omega$. Since the boundary condition is preserved in the limit, we conclude that $u$ belongs to $\mathcal{W}_{\Gamma, u_{0}}\left(\Omega, \mathbb{R}^{3}\right)$.

We now extend by zero $\boldsymbol{n}_{k}$ and $\nabla \boldsymbol{n}_{k}$ to the whole $\mathbb{R}^{3}$. Since the sequence $\left\{\boldsymbol{n}_{k}\right\}$ is bounded in $L^{\infty}$ and the sequence $\left\{\nabla \boldsymbol{n}_{k}\right\}$ is bounded in $L^{2}$, by refining if necessary, we can assume that for certain $l, l^{\prime}$

$$
\lim _{k} \boldsymbol{n}_{k}=l \text { weakly* in } L^{\infty} \text { and } \lim _{k} \nabla \boldsymbol{n}_{k}=l^{\prime} \text { weakly in } L^{2} .
$$

We have to prove that there exists $\boldsymbol{n} \in H^{1}\left([u(\Omega)], \mathbb{S}^{2}\right)$ such that $l=\boldsymbol{n}$ and $l^{\prime}=\nabla \boldsymbol{n}$ on $[u(\Omega)]$. By locality, it is sufficient to prove this in an open neighborhood of each point of $[u(\Omega)]$. Given $\Omega^{\prime} \subset \Omega$, let $x_{0} \in \Omega_{u}^{\prime}$ and $O, O_{k}, r, y_{0}$ as in Lemma 1. Since $B_{r}\left(y_{0}\right)=u_{k}\left(O_{k}\right) \subset\left[u_{k}(\Omega)\right]$, we have $\left\{\left.\boldsymbol{n}_{k}\right|_{B_{r}\left(y_{0}\right)}\right\} \subset H^{1}\left(B_{r}\left(y_{0}\right), \mathbb{R}^{3}\right)$. Therefore $\boldsymbol{n}:=\left.l\right|_{B_{r}\left(y_{0}\right)}$ belongs to $H^{1}\left(B_{r}\left(y_{0}\right), \mathbb{R}^{3}\right)$ and $\nabla \boldsymbol{n}=\left.l^{\prime}\right|_{B_{r}\left(y_{0}\right)}$. By the compact embedding of $H^{1}$ in $L^{2}$, we can also assume that $\boldsymbol{n}_{k} \rightarrow \boldsymbol{n}$ pointwise a.e. in $B_{r}\left(y_{0}\right)$. In particular $\boldsymbol{n} \in \mathbb{S}^{2}$ a.e..

By lower semicontinuity of convex functionals with respect to weak convergence, we have

$$
\underset{k}{\liminf } \mathcal{I}_{\text {nem }}\left(u_{k}, \boldsymbol{n}_{k}\right)=\liminf _{k} \int_{\mathbb{R}^{3}}\left|\nabla \boldsymbol{n}_{k}\right|^{2} \mathrm{~d} y \geq \int_{\mathbb{R}^{3}}\left|l^{\prime}\right|^{2} \mathrm{~d} y \geq \mathcal{I}_{\text {nem }}(u, \boldsymbol{n}) .
$$

It remains to show that $\liminf \operatorname{Ii}_{\text {mec }}\left(u_{k}, \boldsymbol{n}_{k}\right) \geq \mathcal{I}_{\text {mec }}(u, \boldsymbol{n})$. Refining the sequence $\left\{\left(u_{k}, \boldsymbol{n}_{k}\right)\right\}$ we can assume that the liminf is actually a limit: in this way, if necessary, we can further refine the sequence keeping the estimates. In order to avoid the difficulty related to the convergence of the composition $\boldsymbol{n}_{k} \circ u_{k}$, we operate a change of variables and work on the deformed configuration. 
We start with a localization argument. Using the same notation of Lemma 1 , given $\Omega^{\prime} \Subset \Omega, x_{0} \in \Omega_{u}^{\prime}$ and $s \leq r$, we set $U:=w\left(B_{s}\left(y_{0}\right)\right)$ and $U_{k}:=w_{k}\left(B_{s}\left(y_{0}\right)\right)$. For $k \geq k_{0}$ we have

$$
\begin{aligned}
\int_{U_{k}} W_{\text {mec }}\left(\nabla u_{k}(x), \boldsymbol{n}_{k}\left(u_{k}(x)\right)\right) \mathrm{d} x & =\int_{U_{k}} \widetilde{W}\left(V_{\boldsymbol{n}_{k}}^{-1}\left(u_{k}(x)\right) \nabla u_{k}(x)\right) \mathrm{d} x \\
& =\int_{B_{s}\left(y_{0}\right)} \widetilde{W}\left(V_{\boldsymbol{n}_{k}}^{-1}(y)\left(\nabla w_{k}\right)^{-1}(y)\right) \mathrm{d} y \\
& =\int_{B_{s}\left(y_{0}\right)} \widetilde{W}\left(\left(\nabla w_{k} V_{\boldsymbol{n}_{k}}\right)^{-1}(y)\right) \mathrm{d} y
\end{aligned}
$$

and similarly

$$
\int_{U} W_{\mathrm{mec}}(\nabla u(x), \boldsymbol{n}(u(x))) \mathrm{d} x=\int_{B_{s}\left(y_{0}\right)} \widetilde{W}\left(\left(\nabla w V_{\boldsymbol{n}}\right)^{-1}(y)\right) \mathrm{d} y .
$$

Since $\left(\nabla w_{k} V_{\boldsymbol{n}_{k}}\right)^{-1}=\operatorname{cof}\left(\nabla w_{k} V_{\boldsymbol{n}_{k}}\right)^{T}$ and $\operatorname{cof}\left(\nabla w_{k} V_{\boldsymbol{n}_{k}}\right)^{-1}=\left(\nabla w_{k} V_{\boldsymbol{n}_{k}}\right)^{T}$, by using Lemma 2 , the polyconvexity of $\widetilde{W}$, and the semicontinuity of convex functionals, we obtain

$$
\liminf _{k} \int_{U_{k}} W_{\mathrm{mec}}\left(\nabla u_{k}(x), \boldsymbol{n}_{k}\left(u_{k}(x)\right)\right) \mathrm{d} x \geq \int_{U} W_{\mathrm{mec}}(\nabla u(x), \boldsymbol{n}(u(x))) \mathrm{d} x .
$$

We then use a covering argument. For any $x \in \Omega_{u}^{\prime}$, let $w^{x}, w_{k}^{x}: B_{r(x)}(u(x)) \rightarrow \mathbb{R}^{3}$ be the inverse functions of $u, u_{k}$ in a neighborhood of $u(x)$ given by Lemma 1 . Since

$$
\left\{w^{x}\left(B_{s}(u(x))\right): x \in \Omega_{u}^{\prime} \text { and } s \leq r(x)\right\}
$$

is a covering of $\Omega_{u}^{\prime}$ and $\inf \left\{\operatorname{diam}\left(w^{x}\left(B_{s}(u(x))\right)\right): s \leq r(x)\right\}=0$, by Vitali covering theorem (see [3], Thm. 2.2.2), there exists $\left\{\left(x_{j}, s_{j}\right)\right\}_{j \in \mathbb{N}}$ such that, setting $U^{j}:=w^{x_{j}}\left(B_{s_{j}}\left(u\left(x_{j}\right)\right)\right.$ ), the family $\left\{U^{j}\right\}_{j \in \mathbb{N}}$ is a covering of $\Omega^{\prime}$ (up to a set of zero measure) and $\bar{U}^{j} \cap \bar{U}^{i}=\emptyset$ if $j \neq i$. For $\varepsilon \in\left(0, r\left(x_{j}\right)-s_{j}\right)$, let $U^{j, \varepsilon}:=w^{x_{j}}\left(B_{s_{j}+\varepsilon}\left(u\left(x_{j}\right)\right)\right)$. For fixed $h \in \mathbb{N}, i, j \in\{1, \ldots, h\}$, and choosing $\varepsilon$ small enough we have $U^{j, \varepsilon} \cap U^{i, \varepsilon}=\emptyset$ if $j \neq i$. Observe now that, since $u_{k}$ converges to $u$ uniformly in $\Omega^{\prime}$, for $k$ large enough (depending on $\varepsilon$ ) one has $U_{k}^{j}:=w_{k}^{x_{j}}\left(B_{s_{j}}\left(u\left(x_{j}\right)\right)\right) \subset U^{j, \varepsilon}$ so that $U_{k}^{j} \cap U_{k}^{i}=\emptyset$ if $j \neq i$. Indeed, if $z \in U_{k}^{j}$, then

$$
\left|u(z)-u\left(x_{j}\right)\right| \leq\left|u(z)-u_{k}(z)\right|+\left|u_{k}(z)-u\left(x_{j}\right)\right|<s_{j}+\varepsilon
$$

as soon as $\left\|u-u_{k}\right\|_{\infty} \leq \varepsilon$. By the previous localization argument, we have

$$
\begin{aligned}
\lim _{k} \mathcal{I}_{\text {mec }}\left(u_{k}, \boldsymbol{n}_{k}\right) & \geq \liminf _{k} \int_{\bigcup_{j=1}^{h} U_{k}^{j}} W_{\text {mec }}\left(\nabla u_{k}(x), \boldsymbol{n}_{k}\left(u_{k}(x)\right)\right) \mathrm{d} x \\
& \geq \int_{\bigcup_{j=1}^{h} U^{j}} W_{\text {mec }}(\nabla u(x), \boldsymbol{n}(u(x))) \mathrm{d} x .
\end{aligned}
$$

By letting $h$ go to infinity and by invading $\Omega$ with $\Omega^{\prime}$ we conclude the proof.

Acknowledgements. We thank R. Schiattarella for useful suggestions about the openness of sense preserving maps, and S. Hencl for bringing to our attention [5], Example 1. We also acknowledge valuable discussions with P. Bauman and M.C. Calderer. The research of M. Barchiesi has been partially supported by ERC Advanced Grants $226234-A n T e G e F I$ and 2908888-QuaDynEvoPro. He is also grateful to SISSA (Trieste) for the kind hospitality and support during periods when this work was undertaken. The research of A. DeSimone has been partially supported by the ERC Advanced Grant 340685-MicroMotility. 


\section{REFERENCES}

[1] V. Agostiniani and A. DeSimone, Ogden-type energies for nematic elastomers. Int. J. Nonlin. Mech. 47 (2012) $402-412$.

[2] V. Agostiniani, G. Dal Maso and A. DeSimone, Attainment results for nematic elastomers. Proc. Roy. Soc. Edinb. A, in press (2013).

[3] L. Ambrosio and P. Tilli, Topics on analysis in metric spaces. In vol. 25 of Oxford lecture series in mathematics and its applications. Oxford University Press, New York (2004).

[4] J.M. Ball, Convexity conditions and existence theorems in nonlinear elasticity. Arch. Ration. Mech. Anal. 63 (1976) $337-403$.

[5] J.M. Ball, Global invertibility of Sobolev functions and the interpenetration of matter. Proc. Roy. Soc. Edinb. 88A (1981) $315-328$.

[6] M.C. Calderer, C.A. Garavito and C. Luo, Liquid crystal elastomers and phase transitions in rod networks. Preprint arXiv:1303.6220 (2013).

[7] P. Cesana and A. DeSimone, Strain-order coupling in nematic elastomers: equilibrium configurations. Math. Models Methods Appl. Sci. 19 (2009) 601-630.

[8] P.G. Ciarlet, Mathematical elasticity. I. Three-dimensional elasticity. Vol. 20 of Stud. Math. Appl. North-Holland Publishing Co., Amsterdam (1988).

[9] B. Dacorogna, Direct methods in the calculus of variations. Vol. 78 of Appl. Math. Sci., 2nd ed. Springer, Berlin (2008).

[10] A. DeSimone and L. Teresi, Elastic energies for nematic elastomers. Eur. Phys. J. E 29 (2009) 191-204.

[11] I. Fonseca and W. Gangbo, Local invertibility of Sobolev functions. SIAM J. Math. Anal. 26 (1995) $280-304$.

[12] V.M. Gol'dshtein and Y.G. Reshetnyak, Quasiconformal mapping and Sobolev spaces, vol. 54. Kluwer Academic Publishers, Dordrecht, Germany (1990).

[13] D. Henao and C. Mora-Corral, Invertibility and weak continuity of the determinant for the modelling of cavitation and fracture in nonlinear elasticity Arch. Ration. Mech. Anal. 197 (2010) 619-655.

[14] D. Henao and C. Mora-Corral, Fracture surfaces and the regularity of inverses for BV deformations. Arch. Ration. Mech. Anal. 201 (2011) 575-629.

[15] S. Müller, Q. Tang and B.S. Yan, On a new class of elastic deformations not allowing for cavitation. Ann. Inst. Henri Poincaré, Anal. Non Linéaire 11 (1994) 217-243.

[16] M. Warner and E.M. Terentjev, Liquid Crystal Elastomers. Clarendon Press, Oxford (2003). 